\title{
Microscopic Insight to Nonlinear Voltage Dependence of Charge in Carbon-Ionic Liquid Supercapacitors
}

\author{
Aleksandar Y. Mehandzhiyski $\left(\mathbb{D}\right.$, Xuehang Wang $\mathbb{D}^{\mathbb{D}}$, Candy Anquetil-Deck $\mathbb{D}$, De Chen $(\mathbb{D}$, \\ and Brian A. Grimes
} Norwegian University of Science and Technology, Department of Chemical Engineering, Sem Scelandsvei 4,
N-7491 Trondheim, Norway

Correspondence should be addressed to Aleksandar Y. Mehandzhiyski; aleksandar.mehandzhiyski@liu.se and Brian A. Grimes; brian.a.grimes@ntnu.no

Received 25 January 2021; Accepted 14 May 2021; Published 12 June 2021

Copyright ( 2021 Aleksandar Y. Mehandzhiyski et al. Exclusive Licensee Beijing Institute of Technology Press. Distributed under a Creative Commons Attribution License (CC BY 4.0).

The impact of cell voltage on the capacitance of practical electrochemical supercapacitors is a phenomenon observed experimentally, which lacks a solid theoretical explanation. Herein, we provide combined experimental and molecular dynamics investigation of the relation between voltage and capacitance. We have studied this relation in supercapacitor cells comprising of activated carbon material as the active electrode material, and neat ionic liquids (ILs), and a mixture of ILs as the electrolyte. It has been observed that the increase of accumulative charge impacts the conformation and packing of the cations in the anode, which determines its nonlinear behavior with increasing voltage. It has also been shown that for the mixture IL with two types of cations, the contribution of each type of cation to the overall capacitance is highly dependent on the different pore sizes in the system. The smaller tetramethylammonium $\left(\mathrm{TMA}^{+}\right)$shows tendency for more efficient adsorption in the mesopores, while 1-Ethyl-3-methylimidazolium $\left(\mathrm{EMIM}^{+}\right)$is found to be present almost exclusively in the micropores where $\mathrm{TMA}^{+}$is present in small quantities. Such microscopic insights from computer simulations of the molecular phenomena affecting the overall performance in supercapacitors can help to design more efficient electrolytes and devices.

\section{Introduction}

Development of new and more effective electrochemical energy storage, such as high energy and power electrochemical supercapacitors, is in high demand with the increase of renewable energy produced globally [1-3]. Ionic liquids are promising electrolytes of choice for the electrochemical energy storage application due to their large operational voltage. Improving the capacitance of high-voltage supercapacitor in IL electrolyte is an efficient way to achieve higher energy and power density. In different studies, the increase of the applied voltage leads to an increase of the capacitance in general, although the experimental setups differ from each other $[4,5]$. The effect of the applied voltage on the increasing capacitance can be up to $50 \%$ for activated carbon (AC) electrodes and up to four times for singlewall carbon nanotube (SWCNT) [4, 5]. Both linear and nonlinear dependence of capacitance with the voltage has been observed in these studies.
For an ideal electric double layer capacitor [4], the charge increases linearly with the increase of the applied voltage, and therefore, the capacitance should remain constant. However, many parameters, such as the complicated porous structure [5] and defective carbon surface, could lead to a nonlinear relationship between voltage and charge. Recently, we have shown that the more effective ionic packing in the confined pores is a key parameter for increasing the capacitance [6]. Moreover, the interactions between different cations used as a mixture in the same supercapacitor cell, such as 1-Ethyl3-methylimidazolium $\left(\mathrm{EMIM}^{+}\right)$and tetramethylammonium $\left(\mathrm{TMA}^{+}\right)$, can also alter the packing density in the porous electrodes and, in effect, alter the capacitance [7]. The orientation of nonspherically shaped ions $\left(\mathrm{EMIM}^{+}\right)$changes with the applied voltage, which can alter the packing density of the ion on the electrode surface and the capacitance per surface area [8]. Hence, fundamental insight and understanding of the detailed interactions between the electrode surface and the adsorbed ions at different applied potentials are essential 
in order to rationally tailor the porous electrode material and IL composition for improved SC prototypes.

Molecular simulations are often used to provide qualitative and quantitative insights related to molecular scale phenomena to explain the behavior of complex physical and chemical systems. In particular, molecular dynamics (MD) simulations [9-16] have been successfully applied to study and understand the molecular phenomena occurring in supercapacitor systems. The electrodes (both anode and cathode) in SC have been modelled by a single sheet of graphene [9-12], carbon nanotube [13-15], or complex nanoporous carbon material based on experimental observations [16]. The distribution of the ions and the properties of the electrical double layer on the anode and cathode sides have been a common point of discussion in the simulation studies. In all of these systems, neat ionic liquids (IL) [9-16] or IL in a mixture with organic solvent (acetonitrile) [11] have been used as the electrolyte. Shim et al. [11] have also evaluated the effect of acetonitrile mixed with IL on the performance of the supercapacitor, comprising of graphene sheets, and have shown that the capacitance of the pure IL $\left(\mathrm{EMIM}^{+} \mathrm{BF}_{4}^{-}\right)$is higher than the capacitance of a mixture with acetonitrile. However, the interactions between different cations in the electrolyte mixture in the same supercapacitor cell have not been investigated thoroughly [7].

The structure and density of the ions near the electrodes (both positively and negatively charged) represented as a single graphene layer have been extensively investigated [9-11]. The high capacitance of the IL supercapacitors is correlated with the strong and effective screening of the charged electrode surface by the IL [12]. Nanoporous supercapacitors have been modeled by a carbon nanotube (CNT) [13-15] or carbon material having complex geometry based on experimental pore size distributions [12]. Shim and Kim [13] have studied the behavior of ions inside the pores and the capacitance for various diameters of the CNT for the $\mathrm{EMIM}^{+} \mathrm{BF}_{4}^{-}$ionic liquid. Their results [13] suggested that the capacitance of a CNT supercapacitor with a diameter in the range of 0.9-2 nm increases with decreasing CNT diameter. That observation was associated with the different internal solvation exhibited by ions inside the pores. It was also shown that the lack of internal solvation for pore diameters below $0.8 \mathrm{~nm}$ significantly decreases the capacitance. Although many MD studies [9-16] provide valuable information for understanding the molecular phenomena involved in SC systems, they have not focused on the relations between capacitance, charge, and voltage in a SC.

In the current study, both atomistic MD simulations and experiments have been performed to reveal the voltagedependent charge density relation in porous carbon-ionic liquid supercapacitors. The MD simulations were performed in order to obtain the densities and spatial distributions of IL ions inside a negatively charged porous electrode, modelled by a single wall carbon nanotube. The densities and spatial orientation of the ions inside the pores were calculated for two different pores sizes, four different surface charge distributions, and three different IL compositions. We have determined how the spatial orientations and the densities inside the pores influence the relation between volumetric charge density and voltage in various pores and mixtures of ions in electrolytes. The simulations are shown to be qualitatively in agreement with the experimental data. The simulation setup presented here, although exploring an idealistic case study of a real supercapacitor porous electrode, is suitable for studying the ion orientations and densities and their impact on the capacitance in an ionic mixture electrolyte.

\section{Methods and Models}

2.1. Molecular Dynamics Simulations. A simulation box of 500 pairs of 1-Ethyl-3-methylimidazolium $\left(\mathrm{EMIM}^{+}\right)$and tetrafluoroborate $\left(\mathrm{BF}_{4}^{-}\right)$was initially created. In addition, 110 and 205 pairs of tetramethylammonium $\left(\mathrm{TMA}^{+}\right)$and $\mathrm{BF}_{4}^{-}$were added to mimic the IL mixture composition in the experiments. In total, three simulation boxes were created with $0 \mathrm{wt} \% \mathrm{TMA}^{+}$(System 1), $15 \mathrm{wt} \% \mathrm{TMA}^{+}$(System 2), and $25 \mathrm{wt} \% \mathrm{TMA}^{+}$(System 3) diluted in the $\mathrm{EMIM}^{+} \mathrm{BF}_{4}^{-}$ionic liquid (IL). The simulation boxes were initially equilibrated in the isothermal-isobaric statistical (NPT) ensemble at $293.15 \mathrm{~K}$ and $1 \mathrm{bar}$ of pressure, and the densities of the IL were calculated. The density of the neat IL $\left(0 \mathrm{wt} \% \mathrm{TMA}^{+}\right)$ was calculated to be $1293 \mathrm{~kg} / \mathrm{m}^{3}$ which is in very good agreement with the experimental value of $1297 \mathrm{~kg} / \mathrm{m}^{3}$ [17].

Subsequently, we have used these bulk simulation boxes to create the porous electrode simulations. The porous electrode was created by joining a carbon nanotube with two graphene layers as depicted in Figures 1(c)-1(f). Two of the bulk simulation boxes were placed on both sides of the graphene layers. The porous electrode and simulation snapshots were created with the Visual Molecular Dynamics (VMD) package [18]. In this respect, an idealistic case of a true pore in the real porous electrode has been built, which will enable us to examine the spatial and density distributions of the ILs inside micro- and mesopores in order to construct an abstract model of cation adsorption for the SC anode system described in Reference [6]. Two different carbon nanotubes with diameters of $1.085 \mathrm{~nm}$ and $2.169 \mathrm{~nm}$ have been used to represent a general micro- and mesopore, respectively. The length of the pore was $4.919 \mathrm{~nm}$ and was chosen as a compromise between computational efficiency and sufficient space for the ions to migrate inside. The two graphene layers with dimensions of $4.9 \times 4.9 \mathrm{~nm}$ were placed on both ends of the nanotube. The pore was then left to be filled with the ions from the bulk, which was the first equilibration for 500 ps in the NPT ensemble. A second equilibration also in the NPT ensemble was then carried out for $3 \mathrm{~ns}$. This was done in order to achieve the correct bulk value of the density outside the pore when some of the ions migrate into the pore. The pressure coupling, maintained by the Berendsen barostat [19] at 1 bar, was applied semi-isotropically, where the $x y$-dimensions of the simulation box were kept constant and only the $z$-dimension was scaled. The density was then calculated again after the $3 \mathrm{~ns}$ equilibration to assure that the correct bulk density was achieved. After that, a $5 \mathrm{~ns}$ equilibration in the canonical statistical ensemble (NVT) was carried out, which was followed by a $10 \mathrm{~ns}$ production run also in the NVT ensemble. The coordinates 

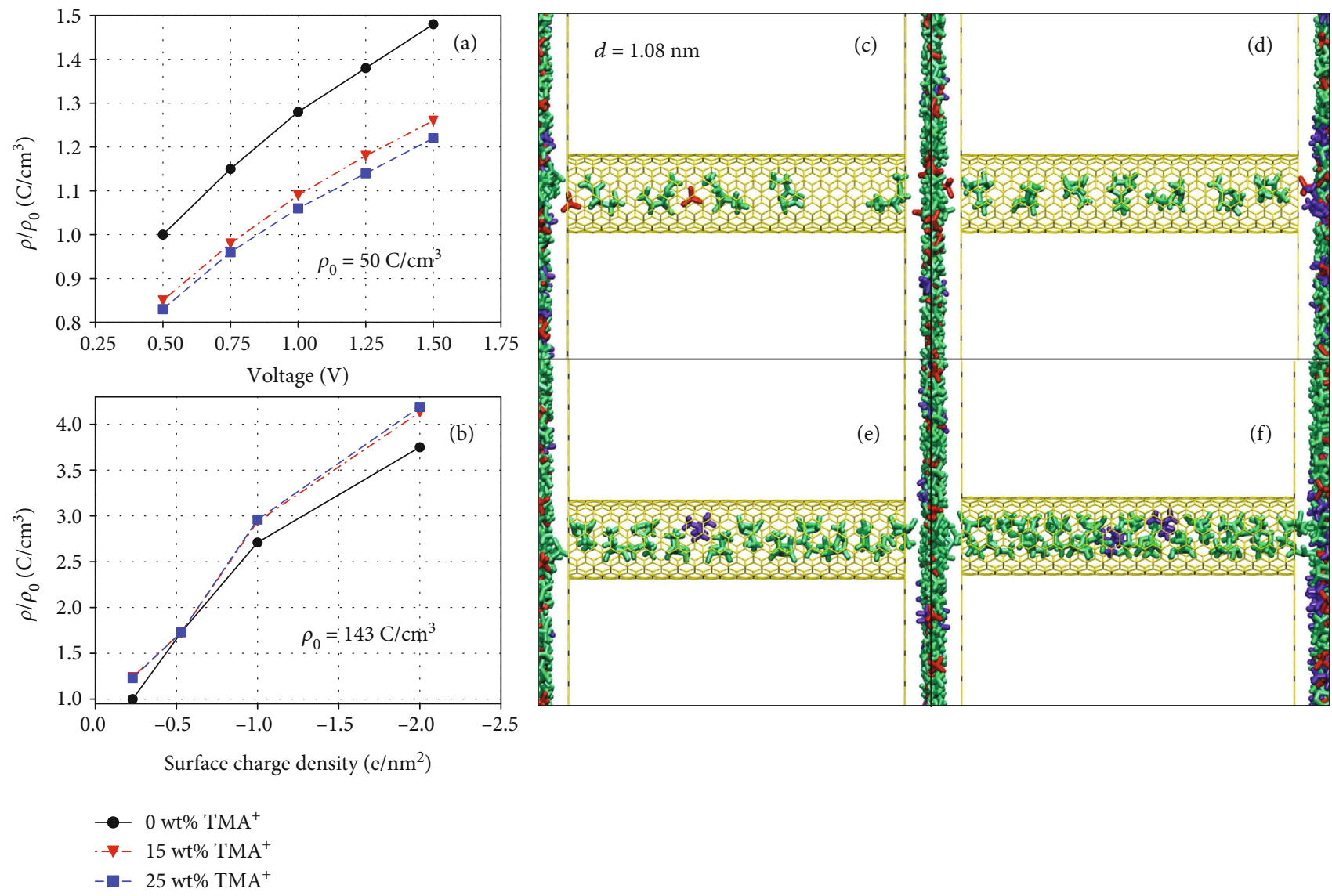

FIGURE 1: Normalized volumetric charge/voltage relation in the micropores obtained experimentally (a) and from the MD simulations (b); simulation snapshots for surface charge densities of $-0.23 \mathrm{e} / \mathrm{nm}^{2}$ (c), $-0.53 \mathrm{e} / \mathrm{nm}^{2}$ (d), $-1.00 \mathrm{e} / \mathrm{nm}^{2}$ (e), and $-2.00 \mathrm{e} / \mathrm{nm}^{2}$ (f). EMIM $\mathrm{EMns}^{+}$are depicted in green, $\mathrm{TMA}^{+}$in blue, and $\mathrm{BF}_{4}^{-}$in red.

of the molecules during the production run were saved every 5 ps.

The nanotubes were negatively charged in order to mimic the applied voltage on the anode in the experimental setup. Four different surface charge distributions $\left(-0.23 \mathrm{e} / \mathrm{nm}^{2}\right.$, $-0.53 \mathrm{e} / \mathrm{nm}^{2},-1.00 \mathrm{e} / \mathrm{nm}^{2}$, and $-2.00 \mathrm{e} / \mathrm{nm}^{2}$ ) were modeled by assigning negative point charges to the nanotube atoms. These surface charge distributions should approximately correspond to $-1.0 \mathrm{~V},-2.0 \mathrm{~V},-4.5 \mathrm{~V}$, and $-9.0 \mathrm{~V}$ applied to the anode, respectively [12]. The numbers of the cations and anions for the different surface charge concentrations of the pore were adjusted in order to keep the overall electroneutrality of the systems. The total charge on the micropore was $-4 e^{-},-9 e^{-},-18 e^{-}$, and $-33 e^{-}$corresponding to the surface charge distributions of $-0.23 \mathrm{e} / \mathrm{nm}^{2},-0.53 \mathrm{e} / \mathrm{nm}^{2},-1.00 \mathrm{e} / \mathrm{nm}^{2}$, and $-2.00 \mathrm{e} / \mathrm{nm}^{2}$, respectively. Therefore, we removed the respective number of $\mathrm{BF}_{4}^{-}$ions to keep the systems electroneutral. In the mesopore systems, we removed $8,18,36$, and 67 anions, respectively. It should be noted that this is a standard procedure found in many simulations $[11,13,15]$.

The $\mathrm{EMIM}^{+}, \mathrm{BF}_{4}^{-}$, and $\mathrm{TMA}^{+}$ions were modelled in full atomistic resolution by using the force field parameters given by Tsuzuki et al. [20]. These force field parameters are slightly modified OPLS-AA parameters for ionic liquids based on those developed by Lopes et al. [21, 22] with the addition of parameters for the $\mathrm{TMA}^{+}$cation. The geometrical combination rule has been used for the nonbonded interactions which are in accordance with the OPLS-AA force field. The temperature was set to $293.15 \mathrm{~K}$ and maintained by the velocity rescaling thermostat [23] with a time constant of $100 \mathrm{fs}$. The time step for integration of the equations of motion was set to $1 \mathrm{fs}$. The electrostatic interactions have been modelled by the Particle-Mesh Ewald summations with a cut-off distance of $1.2 \mathrm{~nm}$. The MD simulations were performed with the GROMACS 5.0.4 simulation package [24], and periodic boundary conditions were applied to all three spatial directions.

The densities, spatial distributions, projected surface area per molecule, and orientation angles of the ions inside the pores were calculated. A molecule is counted to be inside the pore when its center of mass (COM) is inside the nanotube, irrespective of the time spent inside the pore. The projected surface area per molecule was calculated for the $\mathrm{TMA}^{+}$and $\mathrm{EMIM}^{+}$cations, using the Van der Waals molecular surface area projected on a flat surface. Spatial distributions in the radial direction of the nanopores were calculated as follows: the COM of every molecule was calculated first, and then their positions were sorted in shells inside the pores, starting from the pore center, where the shell thickness was $0.1 \AA$. In that way, the probability of finding a 
Table 1: Pore characteristics of carbon materials used in this study.

\begin{tabular}{lcccc}
\hline Sample & $\begin{array}{c}\text { Total pore volume } \\
(0.8 \mathrm{~nm} \text { to } \approx 60 \mathrm{~nm})\left(\mathrm{cm}^{3} / \mathrm{g}\right)\end{array}$ & $\begin{array}{c}\text { Specific surface } \\
\text { area }\left(\mathrm{m}^{2} / \mathrm{g}\right)\end{array}$ & $\begin{array}{c}\text { Micropore volume } \\
(0.8-2 \mathrm{~nm})\left(\mathrm{cm}^{3} / \mathrm{g}\right)\end{array}$ & $\begin{array}{c}\text { Average pore size } \\
(0.8-10 \mathrm{~nm})(\mathrm{nm})\end{array}$ \\
\hline LC600A850 (meso-2.0 $\mathrm{nm})$ & 1.61 & 3140 & 0.73 & 2.00 \\
RC600A850 (meso-1.8 nm) & 2.08 & 4215 & 1.21 & 1.80 \\
RC650A850 (meso-2.7 nm) & 1.48 & 3276 & 0.44 & 2.70 \\
Activated carbon sphere $($ micro-1.3 nm) & 0.49 & 1015 & 0.43 & 1.30 \\
YP-50F AC (micro-1.2 $\mathrm{nm})$ & 0.70 & 1373 & 0.63 & 1.20 \\
TiC-CDC (micro-1.1 $\mathrm{nm})$ & 0.59 & 691 & 0.41 & 1.10 \\
\hline
\end{tabular}

given molecular type in a given shell in the radial direction was obtained, as well as the distance to the nanopore wall. The orientation angles only for the $\mathrm{EMIM}^{+}$cations were calculated, since the other ions are symmetric. Moreover, $\mathrm{EMIM}^{+}$is more flexible compared to $\mathrm{TMA}^{+}$and can adopt different conformations depending on the environment. Therefore, its orientation inside the pores can be influenced by the surface charge.

2.2. Experimental Methods. Three microporous carbon and three mesopore-rich activated carbon electrodes were studied in this work, and all materials' preparation method has been described in detail in our previously published work [7]. Three mesopore-rich carbons were prepared by chemically activating carbonized polyaniline with different carbonization and activation temperatures. Three microporous carbon are commercial activated carbon YP-50, carbide-derived carbon, and activated carbon nanospheres, respectively. Detailed pore characteristics and surface area information of all carbon materials are listed in Table 1. For more detailed information of the material preparation, please see Reference [7].

The carbon materials were separately milled with $5 \mathrm{wt} \%$ Polytetrafluoroethylene (PTFE) solution (Sigma-Aldrich, containing $8.4 \mathrm{wt} \%$ PTFE) in a small amount of ethanol. Afterwards, the mixture of carbon and PTFE was kneaded and rolled into a film and then cut into a disk-shaped electrode with a diameter of $8 \mathrm{~mm}$ and mass of 2.5 to $4 \mathrm{mg}$. The disk-shaped dots were dried in vacuumed oven overnight before being used as active electrode materials for the supercapacitors.

Symmetric configuration of the SCs was used in this study, with two carbon electrodes with the same mass as positive and negative electrodes, respectively. A carboncoated aluminum foil was used as a current collector, while a $25 \mu \mathrm{m}$ thick polypropylene membrane (Celgard 3501) was used as a separator. All cells were fabricated in a glovebox (Vacuum Technologies Inc.) with $\mathrm{O}_{2}<0.1$ ppm and $\mathrm{H}_{2} \mathrm{O}<0.1 \mathrm{ppm}$. After adding 1-Ethyl-3-methylimidazolium tetrafluoroborate $\left(\mathrm{EMIM}^{+} \mathrm{BF}_{4}^{-}\right)$or 15 and 25 $\mathrm{wt} \%$ of tetramethylammonium tetrafluoroborate $\left(\mathrm{TMA}^{+} \mathrm{BF}_{4}^{-}\right)$ in $\mathrm{EMIM}^{+} \mathrm{BF}_{4}^{-}$as electrolyte onto the electrode materials, the cells were sealed and stabilized overnight before testing. The capacitance of a single electrode was calculated from the discharge curve of Galvanostatic charge/discharge based on

$$
C_{\text {sp,electrode }}=\frac{I \Delta t}{m_{\text {total }} V_{\text {cell }}},
$$

where $I$ is the current used during discharging, $m_{\text {total }}$ is the overall mass of the active materials on both electrodes, $\Delta t$ is the discharge time, and $V_{\text {cell }}$ is the voltage change during discharging (excluding the voltage drop).

\section{Results and Discussion}

3.1. Charge/Voltage Relation in Micropores. The results obtained from the MD simulations and the experiments for the voltage/charge relation in the micropores are presented in this section, while the voltage/charge relation in the mesopores is presented in the following section. The capacitance is normalized by the micropore volume of each material to calculate the contribution of capacitance per pore volume $-C_{\mathrm{vol}}$ $\left(\mathrm{F} / \mathrm{cm}^{3}\right)$. Further, the volumetric charge $-\rho\left(\mathrm{C} / \mathrm{cm}^{3}\right)-$ is estimated by the following formula:

$$
\rho=C_{\mathrm{vol}} \times V
$$

where $V$ is the voltage. Similarly, $\rho$ from the MD simulations has been calculated in $\mathrm{C} / \mathrm{cm}^{3}$, where the average number of cations inside the pore per unit of volume has been calculated from the production run. Furthermore, $\rho$ has been normalized with respect to the lowest $\mathrm{TMA}^{+}$concentration case and lowest voltage (surface charge density in the simulations) $\left(\rho_{0}\right)$, for both experimental/simulation results, and referred to as normalized charge density $\left(\rho / \rho_{0}\right)$. In that way, we can compare the trends obtained from the simulations with the experimental results.

Figure 1 presents the voltage/normalized charge relation in the micropores obtained from the experiments (Figure 1(a)) and simulations (Figure 1(b)), as well as simulation snapshots for the different surface charge densities employed in the simulations. The normalized volumetric charge increases when the voltage (or surface charge density) increases, correlating to the filling of the pore with ions. The slope of the curves gradually decreases, which can be observed from both the experimental and simulation result, indicating the capacitance gradually decreases as the potential increases. Figure 1(a) shows that the increase of $\mathrm{TMA}^{+}$ concentration in the experimental system decreases the capacitance in the micropore. On the other hand, the simulation results in Figure 1(b) demonstrate a negligible increase of the normalized charge with the increase of the $\mathrm{TMA}^{+}$concentration, and it is explained in the next paragraph.

Table 2 summarizes the results for the average number of ions in the pores $\left(N_{E}, N_{B}, N_{T}\right)$ and their respective number 


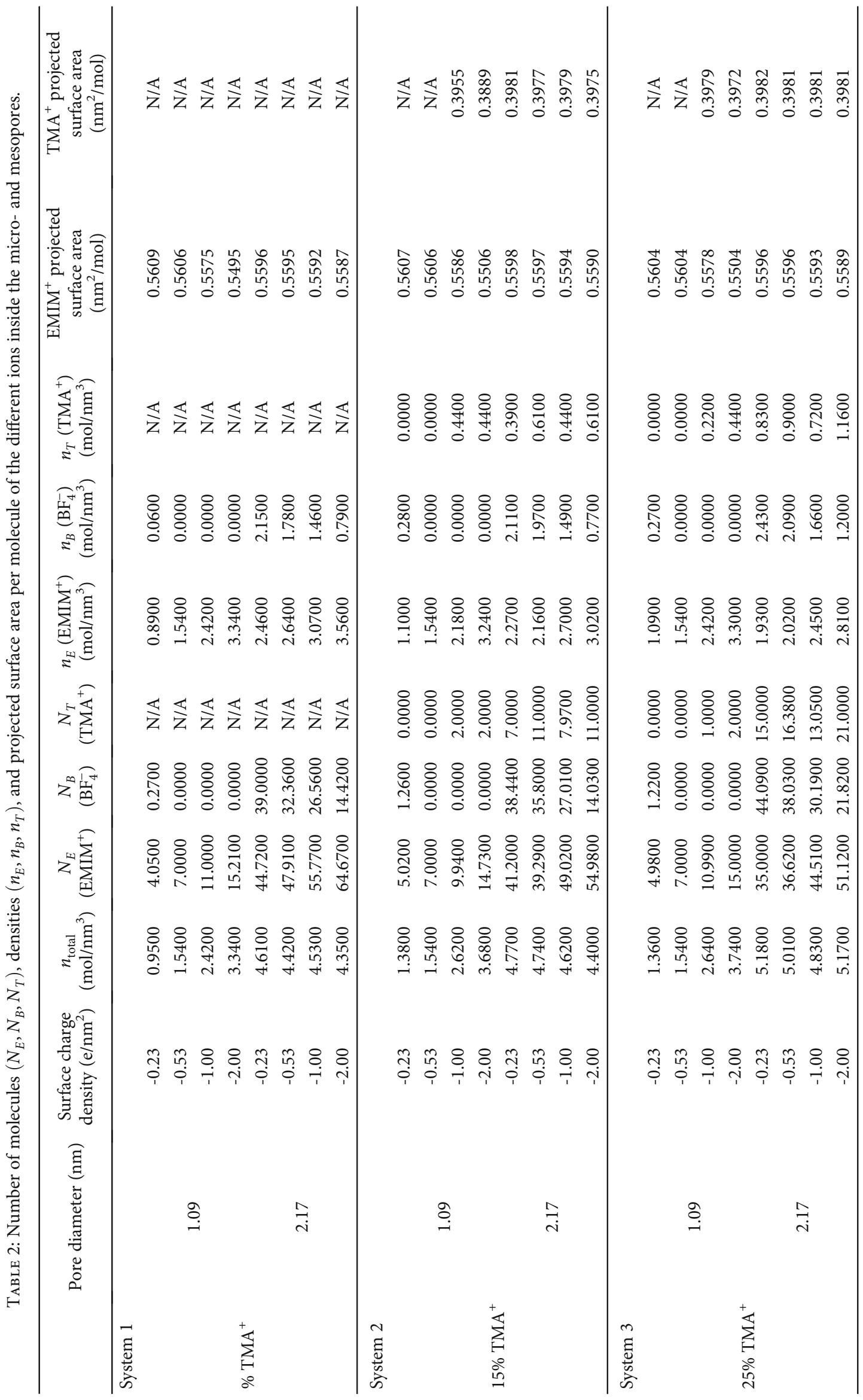


density $\left(n_{E}, n_{B}, n_{T}\right)$ given in molecules $/ \mathrm{nm}^{3}$ for both pore sizes and for all surface charge densities. Figures $1(\mathrm{c})-1(\mathrm{f})$ and Table 2 show that mainly $\mathrm{EMIM}^{+}$cations are present inside the micropore when the surface charge density is low. As the surface charge density increases beyond -1.00 $\mathrm{e} / \mathrm{nm}^{2}, \mathrm{TMA}^{+}$starts to present in minimal quantities in the micropore. The additional $\mathrm{TMA}^{+}$molecules, for surface charge density of $-1.00 \mathrm{e} / \mathrm{nm}^{2}$ and $-2.00 \mathrm{e} / \mathrm{nm}^{2}$, contribute to the increase of the charge density since the surface area $\left(0.4 \mathrm{~nm}^{2} /\right.$ molecule, Table 2) occupied by them is smaller, compared to $\operatorname{EMIM}^{+}\left(0.56 \mathrm{~nm}^{2} /\right.$ molecule, Table 2), and therefore, on average, more molecules can enter the pores. For surface charge density of $-0.50 \mathrm{e} / \mathrm{nm}^{2}$, it can be seen from Figure 1(b) that the volumetric charge density is the same for all three systems studied. This can be explained with the fact that for all systems with this surface charge $\left(-0.50 \mathrm{e} / \mathrm{nm}^{2}\right)$, the same number (7) of $\mathrm{EMIM}^{+}$molecules is found inside the micropores, irrespective of the $\mathrm{TMA}^{+}$concentration. For the lowest surface charge density, also some $\mathrm{BF}_{4}^{-}$molecules are found in the micropore, and they are slightly more on average for systems 2 and $3(\approx 1.2)$ compared with system 1 $(\approx 0.2)$. The effect of this is that more $\mathrm{EMIM}^{+}$molecules are found in system 2 and $3(\approx 5)$ compared with system $1(\approx 4)$, as seen from Table 2 . Thus, the volumetric charge density in systems 2 and 3 is higher compared with that in system 1. Therefore, the increase in the surface charge density for systems 2 and 3 (except for $-0.50 \mathrm{e} / \mathrm{nm}^{2}$, which is identical for all systems) in the simulations is explained with the increase of $\mathrm{BF}_{4}^{-}$and $\mathrm{TMA}^{+}$molecules present in the micropores in these two systems. For the two lowest surface charge densities, no $\mathrm{TMA}^{+}$was found in the micropore, consistent with our previous study [7]. Meanwhile, the $\mathrm{TMA}^{+}$with a more concentrated charge should have a stronger attraction with the electrode (evident also from the radial distributions presented in Section 3.3, where $\mathrm{TMA}^{+}$is found closer and more tightly packed than $\mathrm{EMIM}^{+}$to the pore wall); hence, the $\mathrm{TMA}^{+}$ion transport is more resistive compared with $\mathrm{EMIM}^{+}$. With such a confined space, the presence of the $\mathrm{TMA}^{+}$can easily block the pore, leading to a lower capacitance. This explains the noticeable capacitance decreases when $\mathrm{TMA}^{+}$is added based on the experimental result. Especially when the voltage increase, the excess of the $\mathrm{TMA}^{+}$ion leads to more capacitance loss as shown in Figure 1(a).

3.2. Charge/Voltage Relation in Mesopores. The relation between normalized charge and voltage in the mesopores is presented in Figure 2. Both experiments (Figure 2(a)) and simulations (Figure 2(b)) clearly show that the capacitance increases with the addition of $\mathrm{TMA}^{+}$. The slope of the curve becomes smaller experimentally, indicating capacitance decreases with the increase of the applied voltage. The smaller slope at the high voltage agrees with the observation in the MD simulation for the $0 \mathrm{wt} \%$ and $15 \mathrm{wt} \%$ of the $\mathrm{TMA}^{+}$. Larger ion transport resistance (pore blockage due to stronger interfacial forces, as explained in Section 3.1) at larger potential, may be one explanation of decreasing capacitance. However, an abnormal capacitance increase in the simulations (Figure 2(b)) is observed at large applied voltage for $25 \mathrm{wt} \%$ of the $\mathrm{TMA}^{+}$. This is probably due to an abrupt increase of the ratio of the $\mathrm{TMA}^{+}\left(n_{T} /\left(n_{E}{ }^{+} \times n_{B}{ }^{+} \times n_{T}{ }^{+}\right)\right)$ from $15 \%$ to $22.4 \%$ when the surface charge density increases from $-1.00 \mathrm{e} / \mathrm{nm}^{2}$ to $-2.00 \mathrm{e} / \mathrm{nm}^{2}$.

From the comparison of Figure 1(b) with Figure 2(b), it can be noted that the increase of the normalized charge with voltage is more than twice as effective in the micropores than in the mesopores based on the simulation result. This indicates that the capacitance per pore volume is much larger in the micropore than in the mesopore, or in other words, the utilization of the pore volume by the ions is more efficient in the micropores. This agrees with the experimental results and the literature that the normalized capacitance is higher in the micropores $[6,25]$.

The surface charge effects on the flexibility of $\mathrm{EMIM}^{+}$in the mesopore are expected to be less pronounced due to the screening of the charges by the presence of the $\mathrm{BF}_{4}^{-}$ions in the pore. The presence of $\mathrm{BF}_{4}^{-}$in the mesopore is significant in the lower surface charge cases, since the negatively charged surface is screened by the cations. However, with the increase of the surface charge, the amount of $\mathrm{BF}_{4}^{-}$progressively lowers, as evident from Figures 2(c)-2(f) and Table 2, which leaves more space for cation adsorption. The effects of the surface charge on the density, spatial orientations, and distributions of the ions in both pores are examined in more detail in the next section.

To further study how the voltage affects the capacitance, we calculated the differential capacitance $\left(C_{d}\right)$ as a function of voltage from the MD simulations. The differential capacitance was calculated similar to Reference [26], and details of the calculations can be found in the Supporting Information. It is evident from Figure 3 that with the increase of the voltage the capacitance decreases, as it was also explained above. This is also consistent with previous MD simulations of similar systems [15]. With increasing $\mathrm{TMA}^{+}$concentration, the capacitance increases for both micro- and mesopores in the simulations, which is also apparent from the voltage/normalized charge relation presented in Figures 1(b) and 2(b). Thus, it can be concluded that the addition of $\mathrm{TMA}^{+}$should, in the ideal case scenario explored by the MD simulation, increase the capacitance irrespective of the pore size. However, as shown in Figure 1(a), the addition of $\mathrm{TMA}^{+}$ decreases the charge density inside the micropore leading to the decrease of the capacitance, which is attributed to more resistive $\mathrm{TMA}^{+}$ion transport compared to $\mathrm{EMIM}^{+}$.

3.3. Voltage Effects on the Ion Density, Spatial Distribution, and Orientations in the Micro- and Mesopores. More information can be obtained by examining the number density of the different ions and particularly the number density of the cations in the pores. At the lowest surface charge density, the number density of the cations in the micropore is significantly lower than that in the mesopore. However, with the increase of the surface charge, the difference between the densities of the cations inside the two pores becomes insignificant for all the systems. Therefore, the packing of the molecules inside the micropore becomes more effective with the increase of the surface charge. The higher cation density in the pores for the elevated voltage would imply higher charge density. It should be also noted that the increase of the charge 


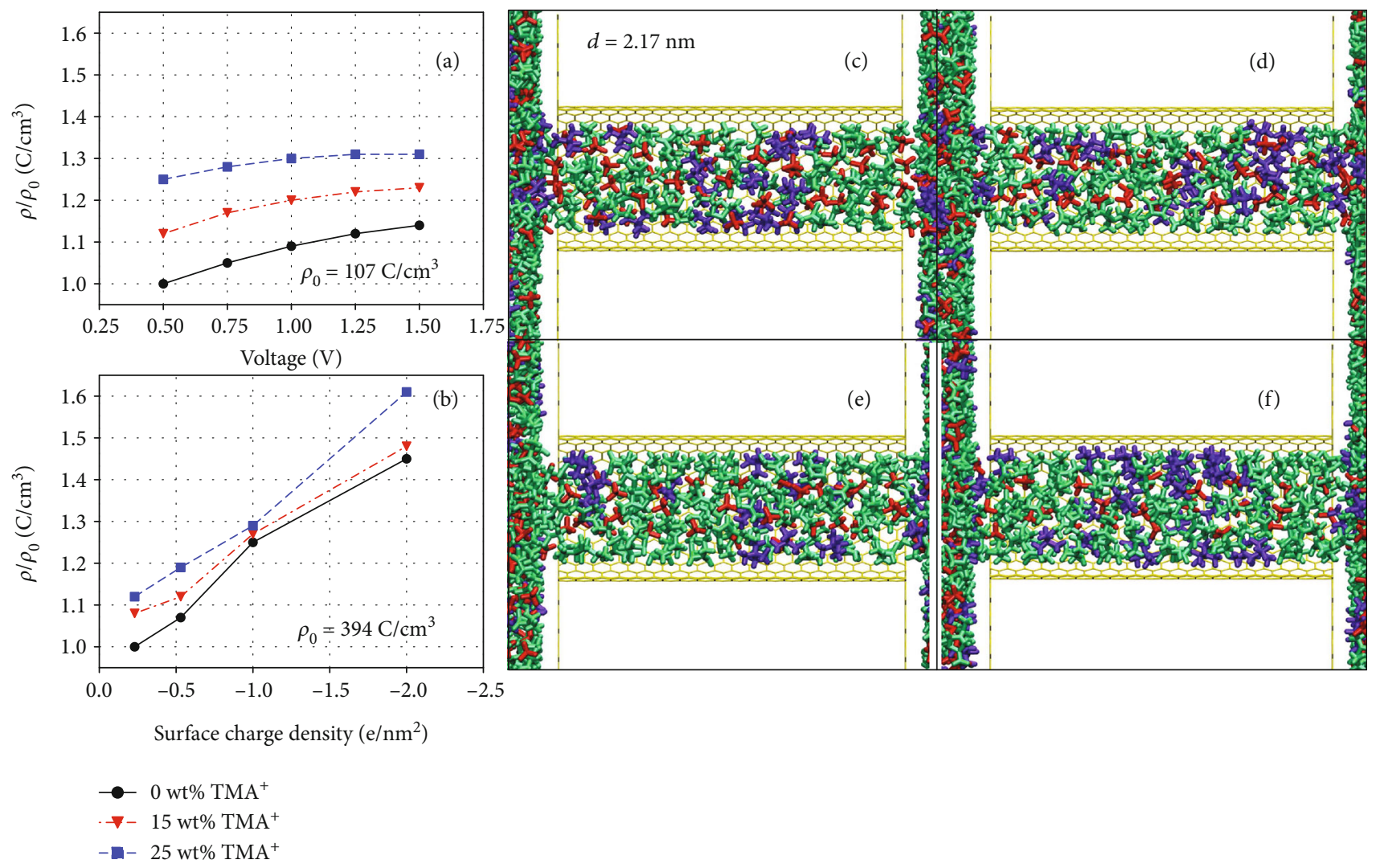

FIGURE 2: Normalized volumetric charge/voltage relation in the mesopores obtained experimentally (a) and from the MD simulations (b); simulation snapshots for surface charge densities of $-0.23 \mathrm{e} / \mathrm{nm}^{2}$ (c), $-0.53 \mathrm{e} / \mathrm{nm}^{2}$ (d), $-1.00 \mathrm{e} / \mathrm{nm}^{2}$ (e), and $-2.00 \mathrm{e} / \mathrm{nm}^{2}$ (f). EMIM ions are depicted in blue, $\mathrm{TMA}^{+}$in green, and $\mathrm{BF}_{4}^{-}$in red.

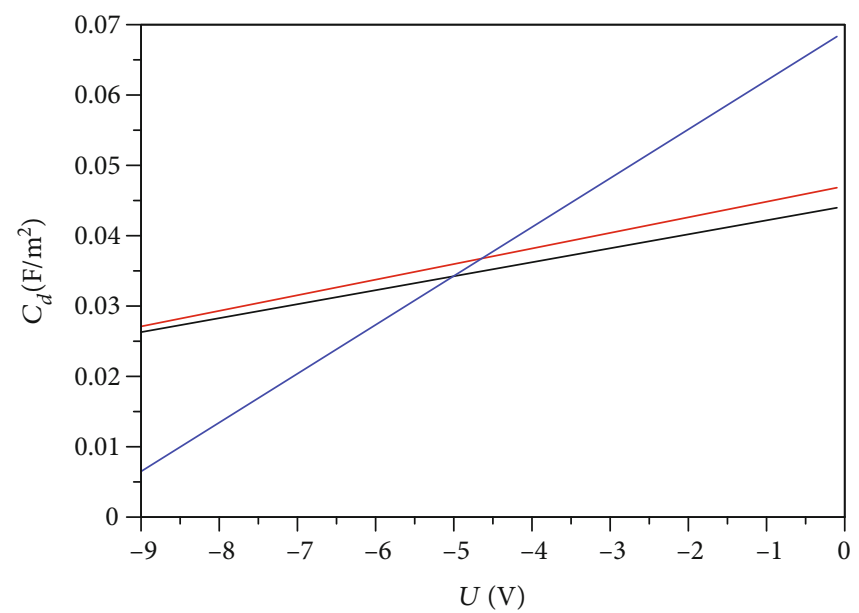

(a)

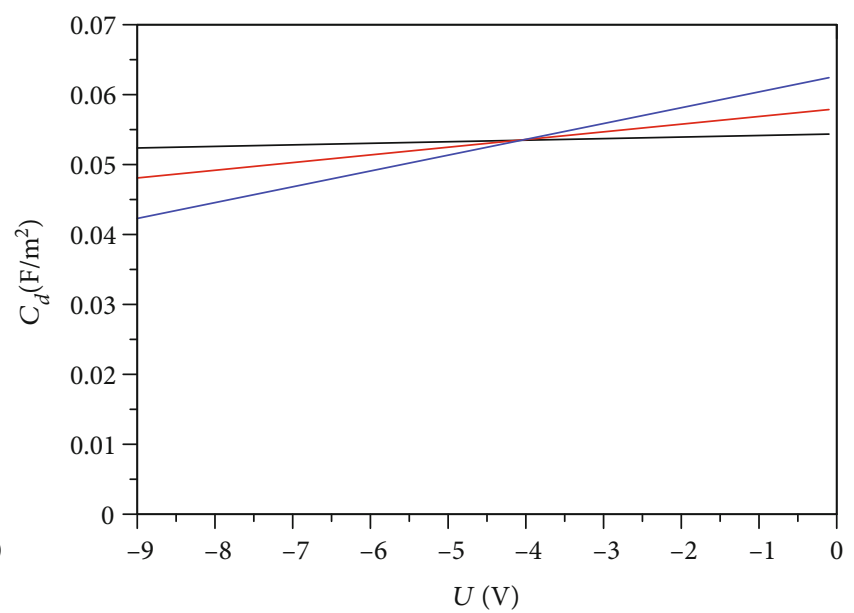

(b)

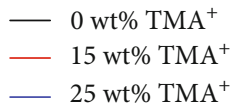

FIGURE 3: Differential capacitance as a function of voltage calculated from the simulations for (a) micropore and (b) mesopore.

density in the micropores comes from the increase of the $\mathrm{EMIM}^{+}$, since $\mathrm{TMA}^{+}$barely presents in the micropore. However, the opposite is true for the mesopores where the increase of the charge density in the mesopores comes from the increased presence of $\mathrm{TMA}^{+}$which also possesses smaller surface area than $\mathrm{EMIM}^{+}$. Therefore, a higher density of the 


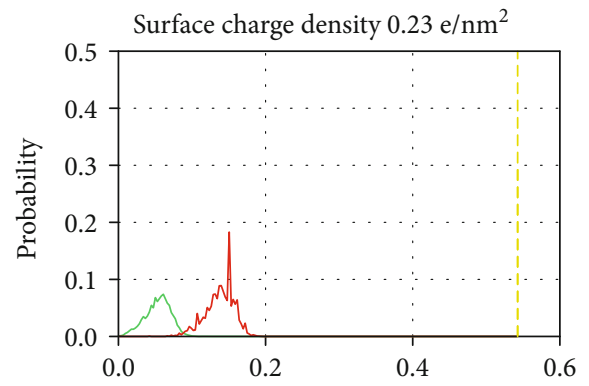

(a)



(c)

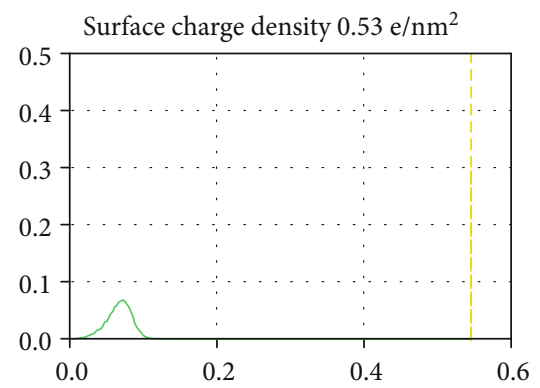

(b)

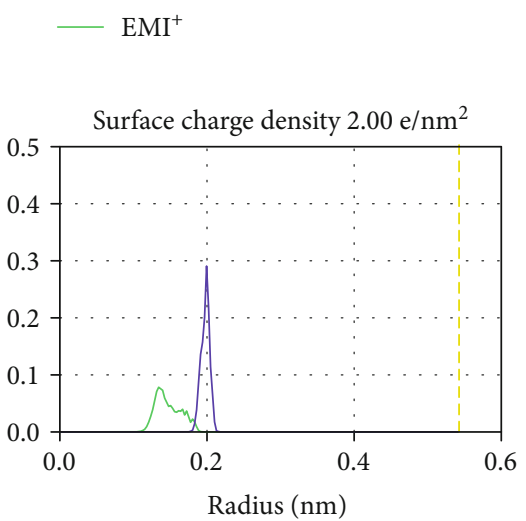

(d)

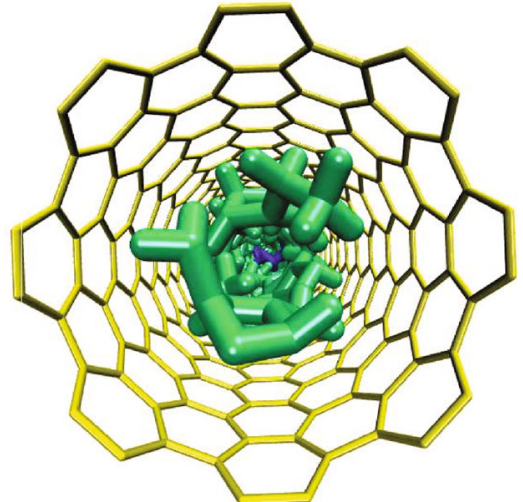

(e)

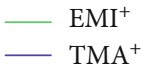

$-\mathrm{EMI}^{+}$

$-\mathrm{TMA}^{+}$

FiguRE 4: Radial distributions of the molecules inside the micropore for system 3 and surface charge densities of (a) -0.23 e/nm ${ }^{2}$, (b) -0.53 $\mathrm{e} / \mathrm{nm}^{2},(\mathrm{c})-1.00 \mathrm{e} / \mathrm{nm}^{2}$, and (d) $-2.00 \mathrm{e} / \mathrm{nm}^{2}$ and (e) view along the axial direction of the nanotube, where EMIM ${ }^{+}$is depicted in green and $\mathrm{TMA}^{+}$in blue.

cations can also be achieved with the increase of $\mathrm{TMA}^{+}$concentration in mesopore systems.

That result is also in agreement with the results shown in Figures 2(a) and 3(b), where the mesopore capacitance increases with the $\mathrm{TMA}^{+}$concentration. It is also apparent from Figure 1(a) that the capacitance in the micropores even decreases with the increase of $\mathrm{TMA}^{+}$concentration. That moreover supports the idea of the contribution of $\mathrm{TMA}^{+}$to the increase of capacitance only in the mesopores and of $\mathrm{EMIM}^{+}$in the micropores. The increase of capacitance in these systems was described also in our previous study [7] and attributed to the selective behavior of the cations towards the micro- and mesopores. The results presented here strongly support our previous observations concerning the selectivity. Additionally, the results presented here suggest that $\mathrm{EMIM}^{+}$and $\mathrm{TMA}^{+}$show preferential adsorption in the micropore and mesopore, respectively. The selectivity behavior of the cations is further discussed in the next section.

Figures 4 and 5 present the positions of the COM of the different molecules inside the micro- and mesopores (system 3 ) along the radial direction of the pores, respectively; here, zero on the $x$-axis represents the middle of the pore, and the probabilities are calculated in the radial direction with respect to the centerline of the pore. The dashed line in the figures represents the pore wall. When the surface charge is low $\left(-0.23 \mathrm{e} / \mathrm{nm}^{2}\right)$ both the cations and anion can be found near the pore wall. The surface charge density is not high enough to repel the anion and could be screened by the cations. When the surface charge density increases, both the $\mathrm{EMIM}^{+}$and $\mathrm{TMA}^{+}$are brought closer to the pore wall, and $\mathrm{TMA}^{+}$is closer to the pore wall than $\mathrm{EMIM}^{+}$for both pore sizes, as seen from Figures 4(c), 4(d), 5(c), and 5(d). Inside the mesopore (Figure 5), we can further observe the formation of three distinctive layers and these layers become more prominent with the increase of the surface charge of the pore.

Figure 6 presents the orientation angle distributions of $\mathrm{EMIM}^{+}$inside the micropore system 1 (Figure 6(a)), micropore system 3 (Figure 6(c)), mesopore system 1 (Figure 6(c)), and mesopore system 3 (Figure 6(d)). The orientation angle is calculated with respect to the axial direction ( $z$-dimension) of the nanotube, where $0^{\circ}$ is parallel to the $z$ -dimension and $90^{\circ}$ angle is perpendicular to the $z$-dimension, as depicted in Figure 6(e), where also the definition of the angle is presented. The orientation angle is defined as the angle between the vector connecting the two carbon atoms located next to the imidazolium ring and the axial direction of the nanotube. The surface charge of the nanotube affects the orientation of $\mathrm{EMIM}^{+}$significantly. When the surface charge is low, below $1.00 \mathrm{e} / \mathrm{nm}^{2}$, the orientation has a broader peak centered between $40^{\circ}$ and $60^{\circ}$, whereas, 


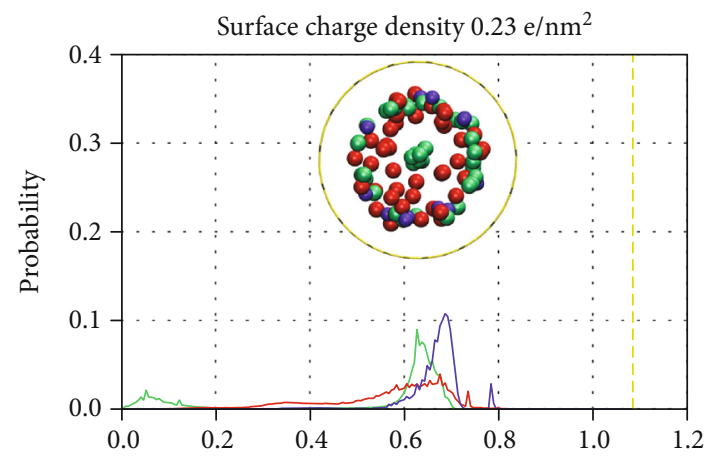

(a)
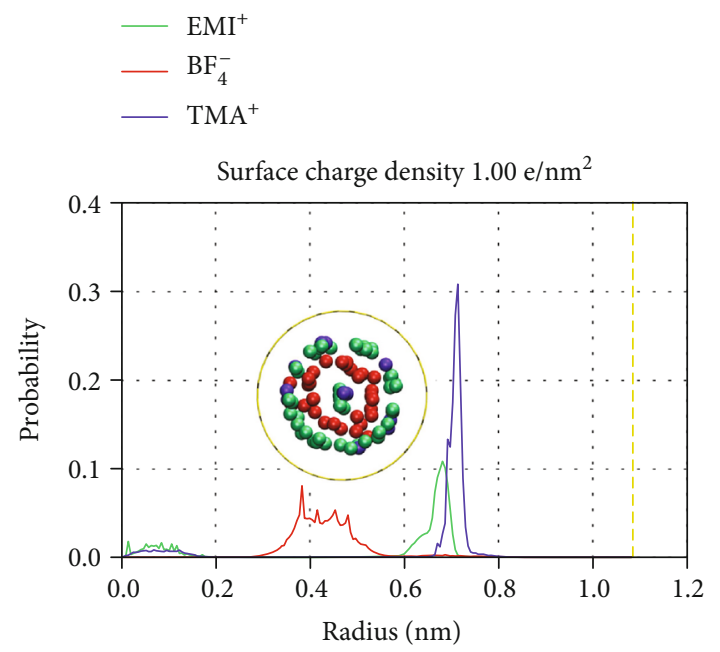

(c)

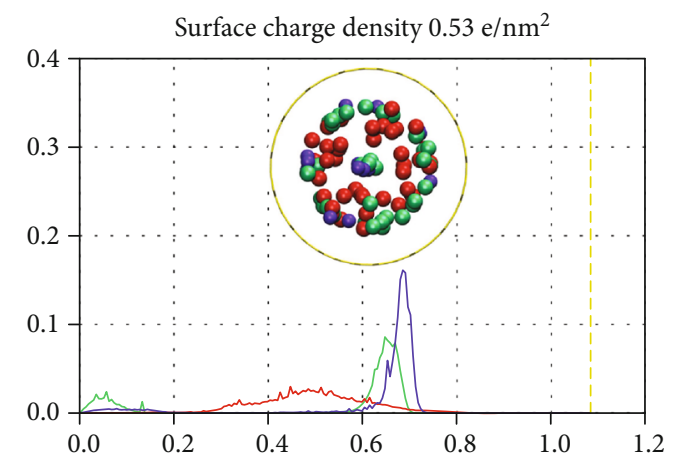

(b)
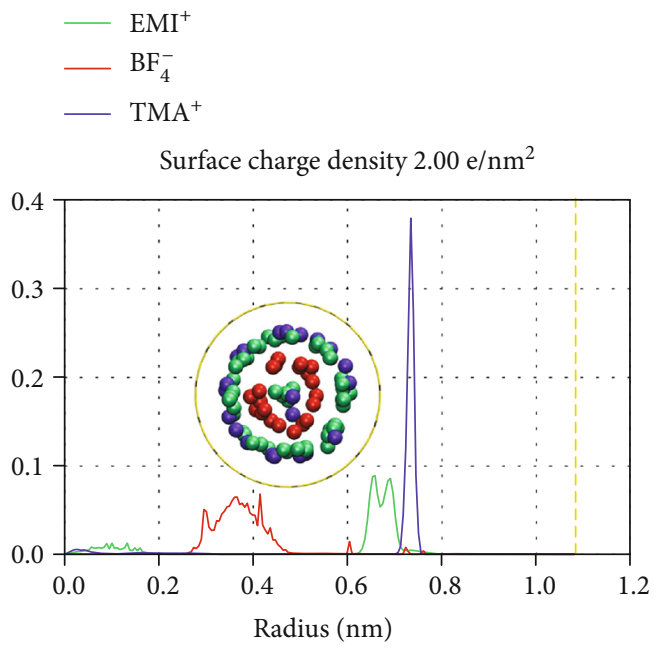

(d)
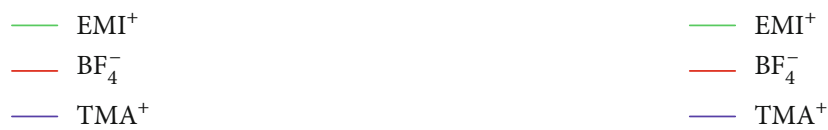

FIGURE 5: Radial distributions of the molecules inside the mesopore for system 3 and surface charge densities of (a) -0.23 e/nm ${ }^{2},(b)-0.53$ $\mathrm{e} / \mathrm{nm}^{2}$, (c) $-1.00 \mathrm{e} / \mathrm{nm}^{2}$, and (d) $-2.00 \mathrm{e} / \mathrm{nm}^{2}$. The insets show the view along the axial direction of the nanotube, where EMIM ${ }^{+}$is depicted in green, $\mathrm{TMA}^{+}$in blue, and $\mathrm{BF}_{4}^{-}$in red. The spheres represent the COM of the respective molecules.

for surface charge of $1.00 \mathrm{e} / \mathrm{nm}^{2}$ and above, several peaks appear where the most dominant peak is close to $90^{\circ}$. The peaks are also narrower compared to the peaks with the lower surface charge. This indicates also that $\mathrm{EMIM}^{+}$is almost locked in this configuration and a rearrangement of the molecules inside the micropore is difficult. Thus, the high surface charge has a solidifying effect on the molecules; i.e., they are close to a solid-state form as the surface charge increases. As seen also from Figure 6(g), $\mathrm{EMIM}^{+}$is quite flexible, allowing it to obtain configurations which follow the curvature of the micropore. Considering that our simulations do not include polarization effects, the dependence of $\mathrm{EMIM}^{+}$configuration of the surface charge is clearly seen. The orientation of $\mathrm{EMIM}^{+}$in the mesopores (Figures 6(c) and 6(d)) is slightly different, because of the presence of $\mathrm{BF}_{4}^{-}$and higher concentrations of $\mathrm{TMA}^{+}$. It seems that the presence of $\mathrm{TMA}^{+}$shifts the orientation angle of $\mathrm{EMIM}^{+}$towards $90^{\circ}$. Also, with the increase of the surface charge, the angle shifts to $90^{\circ}$ in the mesopore, but to a lesser extent.
3.4. Selectivity Behavior and Free Energy of Adsorption. In our previous study, we have observed a selective behavior of the two cations towards entering the micro and mesopores [7]. It was shown that $\mathrm{TMA}^{+}$was able to enter only the mesopores, except when a higher surface charge was applied on the micropore. That was attributed to the stronger interactions of $\mathrm{TMA}^{+}$with $\mathrm{BF}_{4}^{-}$in the bulk, which promotes more difficult dissociation of the cation-anion complex. In this section, we further explore the selectivity behavior by calculating the radial distribution functions (RDF) of $\mathrm{EMIM}^{+}-\mathrm{BF}_{4}^{-}$and $\mathrm{TMA}^{+}-\mathrm{BF}_{4}^{-}$(Figure 7) in the bulk and the respective free energy of adsorption into the pores of $\mathrm{EMIM}^{+}$and $\mathrm{TMA}^{+}$ (Figure 8).

The RDFs presented in Figure 7(a) show that the interaction between $\mathrm{TMA}^{+}$and $\mathrm{BF}_{4}^{-}$is much stronger than the interaction between $\mathrm{EMIM}^{+}$and $\mathrm{BF}_{4}^{-}$. It is also apparent, from the decreasing of the first peak in the RDF for the different systems, that the interaction between $\mathrm{EMIM}^{+}$and $\mathrm{BF}_{4}^{-}$becomes slightly weaker with the addition of $\mathrm{TMA}^{+}$. The first peak of 


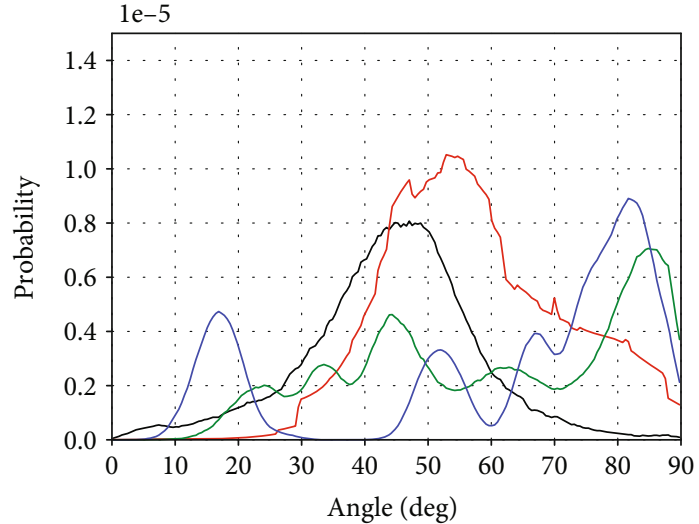

(a) Micropore
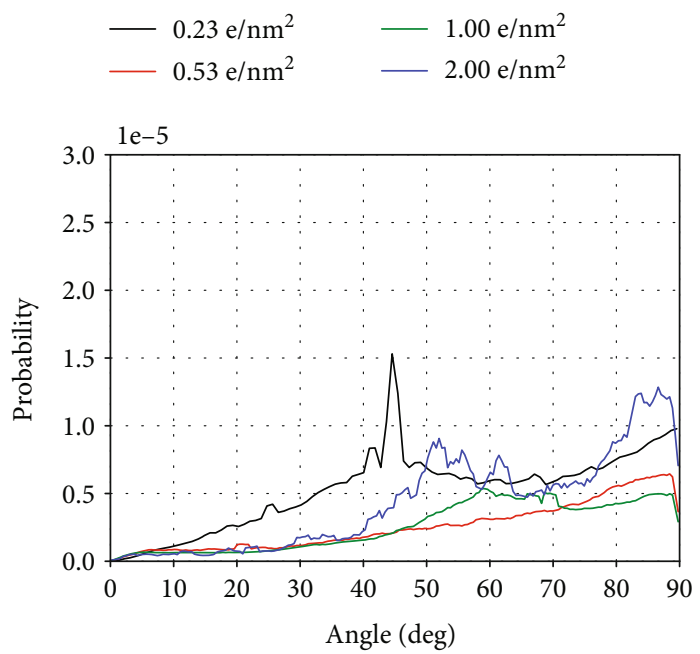

(c) Mesopore

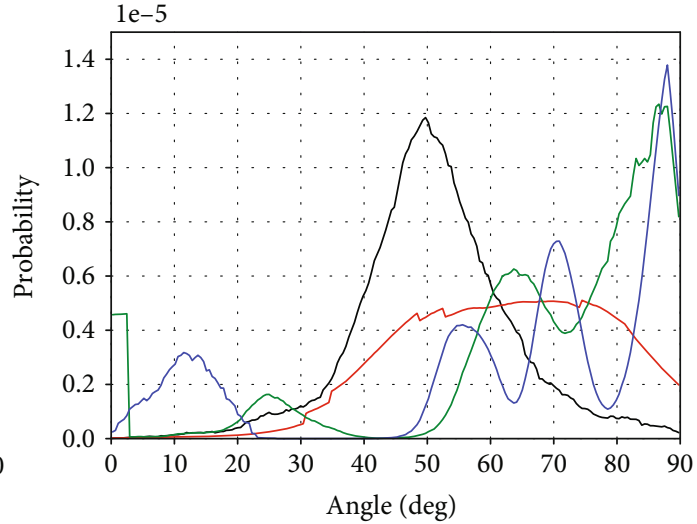

(b) Micropore
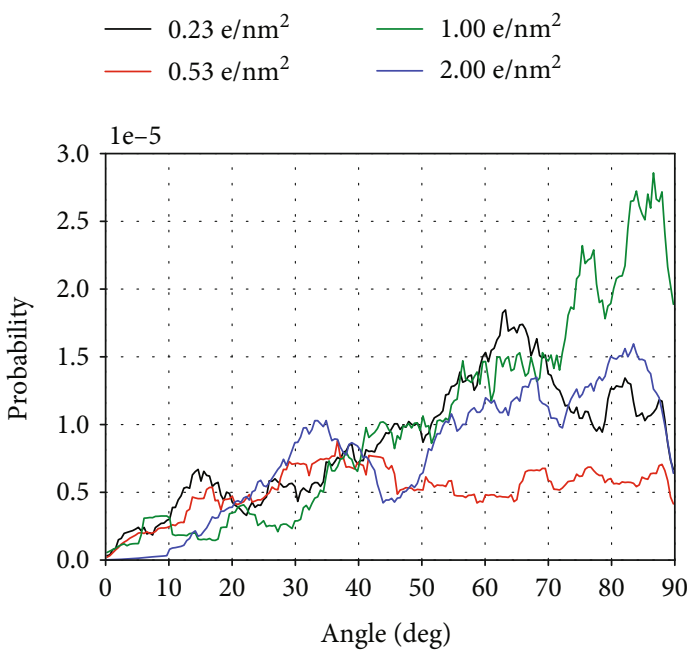

(d) Mesopore

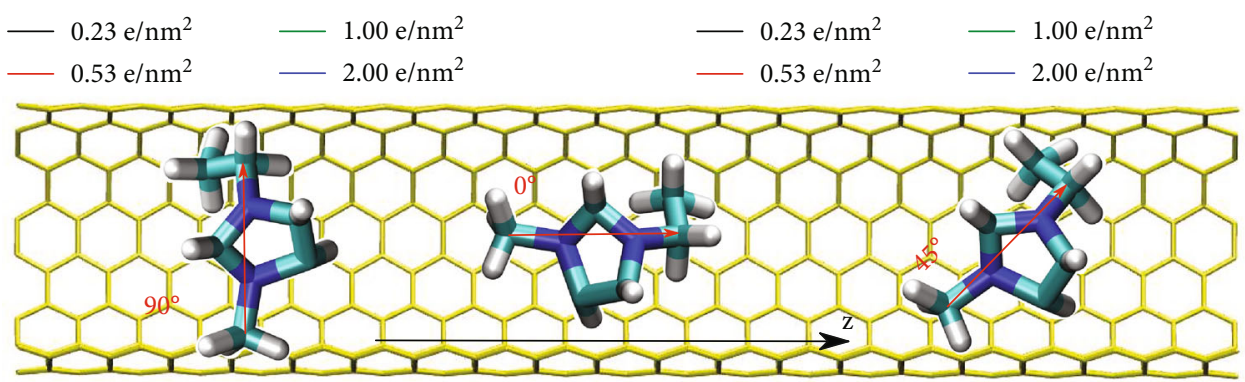

(e)

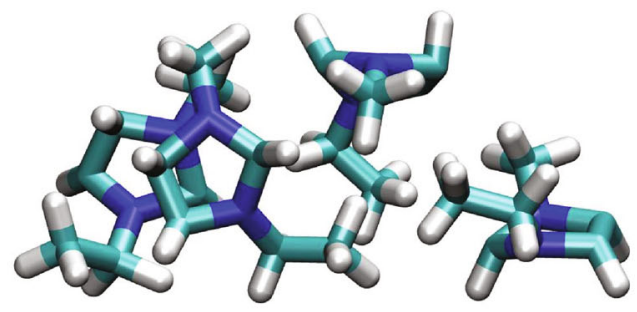

(f)

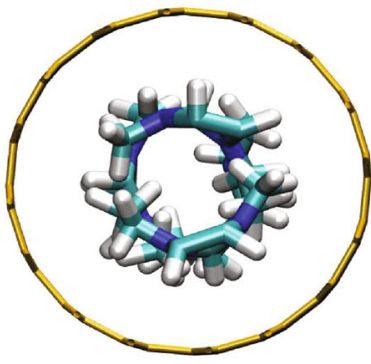

(g)

FIgure 6: Angle orientation distributions along the axial direction of the micropore for (a) $0 \mathrm{wt} \% \mathrm{TMA}^{+}$(system 1) and (b) $25 \mathrm{wt}^{\mathrm{a}} \mathrm{TMA} \mathrm{TM}^{+}$ (system 3). Angle orientation distributions along the axial direction of the mesopore for (c) $0 \mathrm{wt} \% \mathrm{TMA}^{+}$(system 1 ) and (d) $25 \mathrm{wt} \%$ $\mathrm{TMA}^{+}$(system 3). (e) Definition of the orientation angle of EMIM ${ }^{+}$with example orientation angle snapshots. (f, e) Simulation snapshots showing the packing of $\mathrm{EMIM}^{+}$in the micropore. 


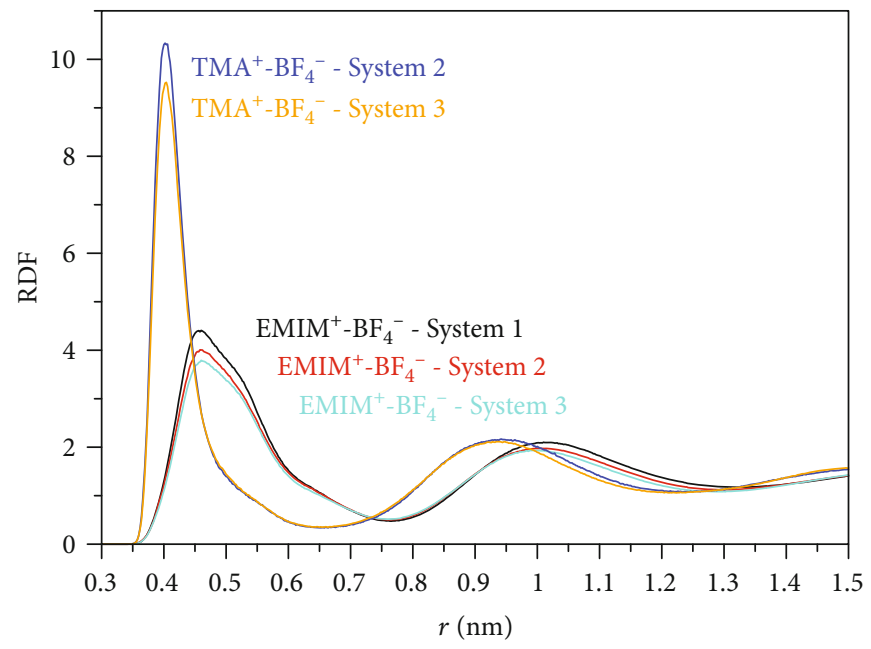

(a)

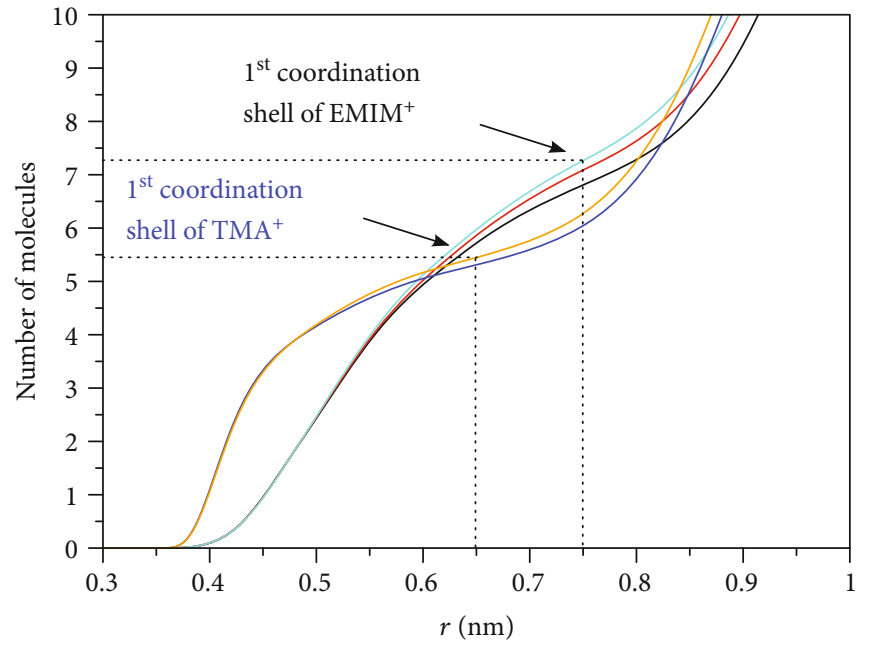

(b)

Figure 7: (a) Radial distribution functions of $\mathrm{EMIM}^{+}-\mathrm{BF}_{4}^{-}$and $\mathrm{TMA}^{+}-\mathrm{BF}_{4}^{-}$and (b) cumulative number RDF corresponding to the distributions in (a).

the RDF also reveals that the distance between $\mathrm{TMA}^{+}$and $\mathrm{BF}_{4}^{-}$is around $0.4 \mathrm{~nm}$, while the distance between $\mathrm{EMIM}^{+}$ and $\mathrm{BF}_{4}^{-}$is around $0.5 \mathrm{~nm}$. This peak is also narrower for $\mathrm{TMA}^{+}-\mathrm{BF}_{4}^{-}$compared with $\mathrm{EMIM}^{+}-\mathrm{BF}_{4}^{-}$, which indicates the formation of more compact and more tightly bound first coordination shell around $\mathrm{TMA}^{+}$. As seen from Figure 7(b), $\mathrm{TMA}^{+}$is able to coordinate around $5 \mathrm{BF}_{4}^{-}$molecules while $\mathrm{EMIM}^{+}$coordinates around $7 \mathrm{BF}_{4}^{-}$molecules, which also indicates more loosely bound first coordination shell in the case of $\mathrm{EMIM}^{+}$. Therefore, the stronger interaction between $\mathrm{TMA}^{+}$and $\mathrm{BF}_{4}^{-}$is likely the dominant factor that dictates the selective adsorption of the cations. $\mathrm{TMA}^{+}$can not enter the micropore, because of the strong interaction with $\mathrm{BF}_{4}^{-}$, and also, their complex is large enough to prevent the adsorption in the micropore. However, when the surface charge increases beyond some threshold, the Coulombic interactions of $\mathrm{TMA}^{+}$with the nanotube become stronger and are able to break the complex with $\mathrm{BF}_{4}^{-}$, and some $\mathrm{TMA}^{+}$cations are able to adsorb into the micropore. The adsorption in the mesopore, however, has a different mechanism. The dimensions of the mesopore are large enough to accommodate the complex of $\mathrm{TMA}^{+}$and $\mathrm{BF}_{4}^{-}$which most likely are dragged together into the pore.

Here, we have also calculated the energy required for a cation to enter the micropore, which effectively is the energy penalty to move from the bulk to the pore. To wit, we have calculated the free energy needed for a cation to enter the micropore which is already filled by other ions from the electrolyte, thus obtaining the insertion free energy of a cation in the pore. This has been done by umbrella sampling simulations [27-30], and the free energy has been calculated by the Weighted Histogram Analysis Method (WHAM) [31, 32]. The umbrella sampling procedure has been applied for system 3 , for surface charge of $-0.53 \mathrm{e} / \mathrm{nm}^{2}$ and $-2.00 \mathrm{e} / \mathrm{nm}^{2}$ and for both $\mathrm{TMA}^{+}$and $\mathrm{EMIM}^{+}$cations. Either $\mathrm{TMA}^{+}$or $\mathrm{EMIM}^{+}$cations have been pulled from the bulk of the box in the direction towards the pore $(z$-dimension in the simu- lation box). The reaction coordinate for calculating the insertion free energy has been defined from the initial position of the chosen ion to the center of the pore. On average, 40 umbrella windows separated by $0.1 \mathrm{~nm}$ have been used to calculate the insertion free energy.

We have calculated the following free energies (Table 3): $\operatorname{EMIM}^{+}\left(-0.53 \mathrm{e} / \mathrm{nm}^{2}-25 \mathrm{kcal} / \mathrm{mol}\right), \mathrm{EMIM}^{+}\left(-2.00 \mathrm{e} / \mathrm{nm}^{2}-\right.$ $45 \mathrm{kcal} / \mathrm{mol}), \mathrm{TMA}^{+}\left(-0.53 \mathrm{e} / \mathrm{nm}^{2}-60 \mathrm{kcal} / \mathrm{mol}\right)$, and $\mathrm{TMA}^{+}$ $\left(-2.00 \mathrm{e} / \mathrm{nm}^{2}-50 \mathrm{kcal} / \mathrm{mol}\right)$. The respective free energy profiles have been presented in Figures 8(a) and 8(b) for surface charge density of $-0.53 \mathrm{e} / \mathrm{nm}^{2}$ and $-2.00 \mathrm{e} / \mathrm{nm}^{2}$, respectively. It is important to note, in the simulations with a surface charge density of $-0.53 \mathrm{e} / \mathrm{nm}^{2}$, no $\mathrm{TMA}^{+}$molecules are observed to enter the micropore, and for $-2.00 \mathrm{e} / \mathrm{nm}^{2}$, some $\mathrm{TMA}^{+}$molecules have entered the micropore during the nonumbrella sampling simulations presented in Section 3.1. Therefore, we have chosen these two cases as examples when $\mathrm{TMA}^{+}$is able or not able to enter the micropore and have calculated the energy needed for the molecules to enter the micropore. That should provide information about which molecule is more energetically favorable to enter the micropore. At the lower surface charge density of $-0.53 \mathrm{e} / \mathrm{nm}^{2}$ in Figure $8(\mathrm{a})$, the energy of $\mathrm{TMA}^{+}$near the beginning of the micropore is higher than the energy of $\mathrm{EMIM}^{+}$indicating that it is favorable for $\mathrm{EMIM}^{+}$to enter the micropore. At the higher surface charge density $\left(-2.00 \mathrm{e} / \mathrm{nm}^{2}\right)$, the energy of the two molecules is nearly the same at the place where the micropore begins. The insertion of $\mathrm{EMIM}^{+}$in this case is still more favorable, but the energy difference between $\mathrm{EMIM}^{+}$and $\mathrm{TMA}^{+}$is only $5 \mathrm{kcal} / \mathrm{mol}$. As it was already shown in Table 2 and Figures 1 and 4 , some $\mathrm{TMA}^{+}$molecules are able to enter the micropore for the higher surface charge densities. It should be noted that the free energy of adsorption of $\mathrm{TMA}^{+}$decreases with the increase of the surface charge density while the opposite is observed for $\mathrm{EMIM}^{+}$. The former could be explained with the stronger attraction of $\mathrm{TMA}^{+}$to the negatively charged pore wall than $\mathrm{EMIM}^{+}$because of the higher charge density 


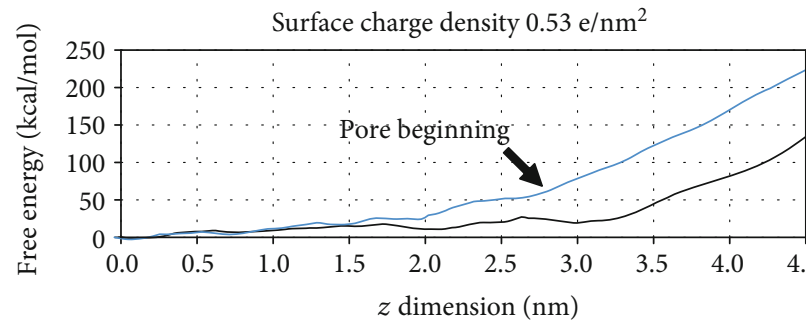

(a)

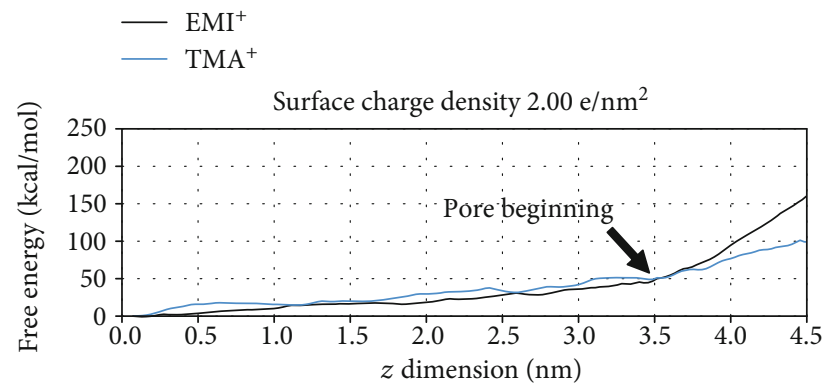

(b)

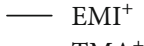

$-\mathrm{TMA}^{+}$

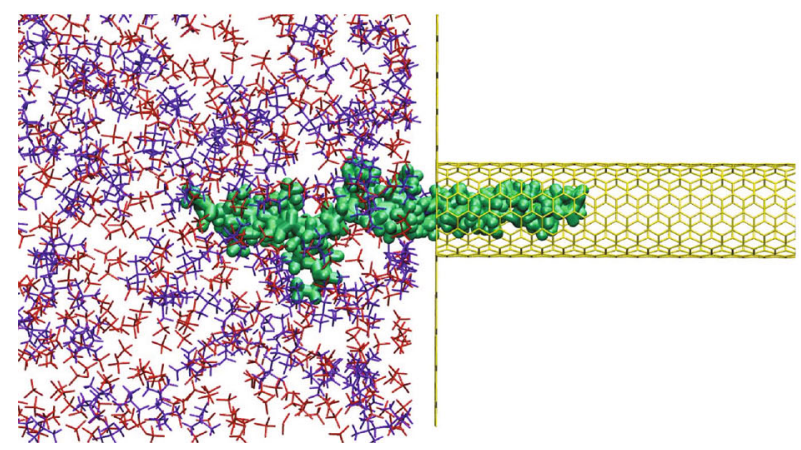

(d)

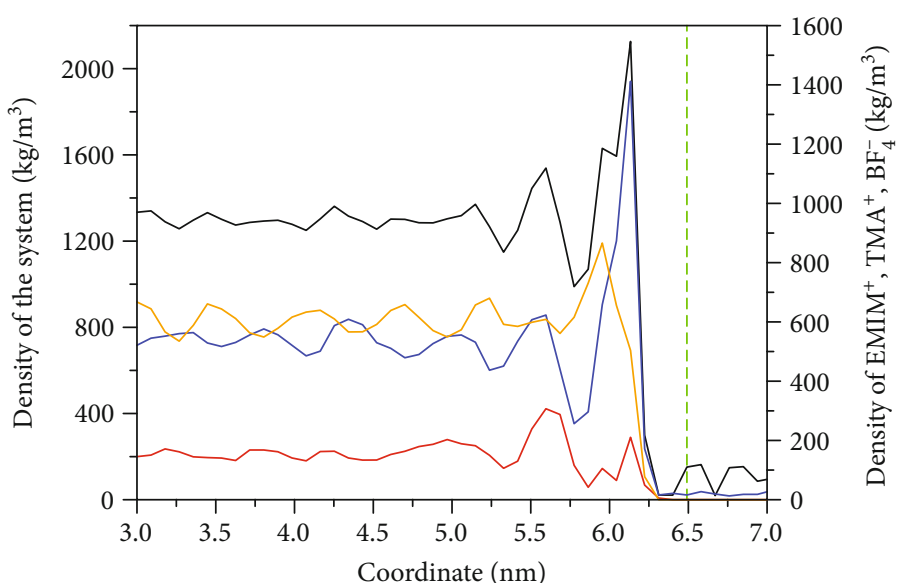

(c)
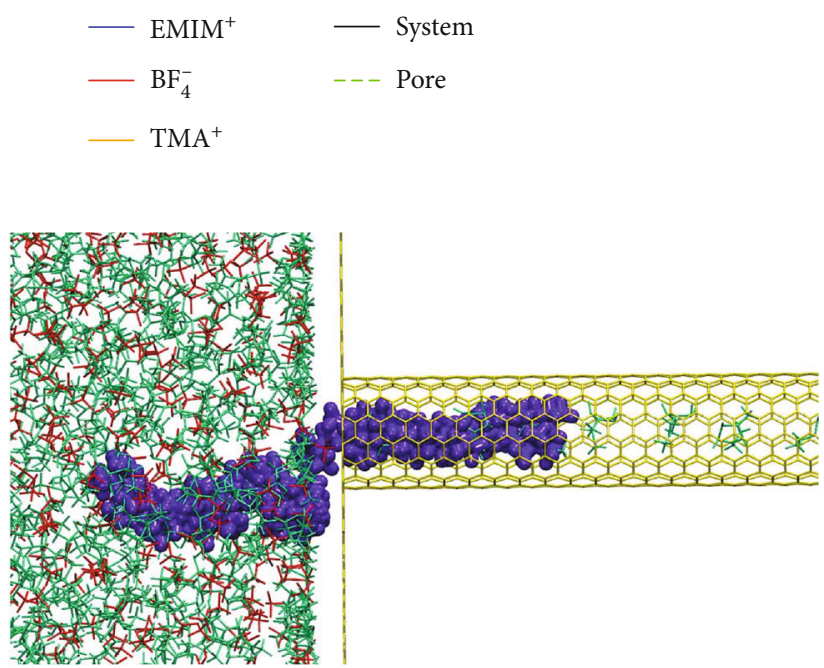

(e)

FIgURE 8: Umbrella sampling simulations: (a) free energy of insertion of $\mathrm{EMIM}^{+}$(black) and $\mathrm{TMA}^{+}$(blue) in the micropore with surface charge density of $-0.53 \mathrm{e} / \mathrm{nm}^{2}$; (b) free energy of insertion of $\mathrm{EMIM}^{+}$(black) and $\mathrm{TMA}^{+}$(blue) in the micropore with surface charge density of $-2.00 \mathrm{e} / \mathrm{nm}^{2}$; (c) density of the system and the different ions in the system in the $z$-dimension of the simulation box; (d) trajectory showing the path of $\mathrm{EMIM}^{+}$(green) entering the micropore; (e) trajectory showing the path of $\mathrm{TMA}^{+}$(blue) entering the micropore. $\mathrm{EMIM}^{+}$molecules are depicted in green, $\mathrm{TMA}^{+}$in blue, and $\mathrm{BF}_{4}^{-}$in red.

TABLE 3: Adsorption free energies of $\mathrm{EMIM}^{+}$and $\mathrm{TMA}^{+}$in the micropore for different surface charge densities.

\begin{tabular}{lll}
\hline & $-0.53 \mathrm{e} / \mathrm{nm}^{2}$ & $-2.00 \mathrm{e} / \mathrm{nm}^{2}$ \\
\hline $\mathrm{EMIM}^{+}$ & $25 \mathrm{kcal} / \mathrm{mol}$ & $45 \mathrm{kcal} / \mathrm{mol}$ \\
$\mathrm{TMA}^{+}$ & $60 \mathrm{kcal} / \mathrm{mol}$ & $50 \mathrm{kcal} / \mathrm{mol}$ \\
\hline
\end{tabular}

of the smaller ion $\left(\mathrm{TMA}^{+}\right)$. Smaller ions with higher charge density interact stronger than large ions. Stronger attraction of $\mathrm{TMA}^{+}$to the pore wall is also evident from the radial distribution presented in Figures 4 and 5, where $\mathrm{TMA}^{+}$is located closer to the wall. Therefore, the increase of the surface charge density favors the adsorption of the smaller cation. The latter, the increase of $\mathrm{EMIM}^{+}$adsorption free energy, we speculate arises from competition of $\mathrm{EMIM}^{+}$for adsorption with $\mathrm{TMA}^{+}$.

From the umbrella sampling simulations, it can be also seen that the adsorption of $\mathrm{EMIM}^{+}$and $\mathrm{TMA}^{+}$is an endothermic process. We speculate that this is due to the formation of an electric double layer near the graphene surface as can be seen from the density plot presented in Figure 8(c) for the case of $-2.00 \mathrm{e} / \mathrm{nm}^{2}$ surface charge. Therefore, the cation needs energy to penetrate a region with an enhanced density, which makes the process endothermic. Figures $8(\mathrm{~d})$ and 8(e) also present the trajectory of $\mathrm{EMIM}^{+}$and $\mathrm{TMA}^{+}$, respectively, to migrate from the bulk into the pore. From these trajectories, it can be observed that $\mathrm{TMA}^{+}$spends relatively more time near the graphene surface than $\mathrm{EMIM}^{+}$. $\mathrm{EMIM}^{+}$ enters the micropore directly probably because it can also more easily change its conformation in the denser double 
layer near the graphene wall outside the pore, than the more rigid $\mathrm{TMA}^{+}$. Another possible factor that can hinder the adsorption of $\mathrm{TMA}^{+}$is its stronger complexation with $\mathrm{BF}_{4}^{-}$ molecules (seen from the RDFs presented in Figure 7(a)) in the double layer region near the pore.

\section{Conclusions}

The relation between the applied voltage and volumetric charge density in a supercapacitor system has been investigated. The experimental results showing a decrease in capacitance with an increase of the applied voltage are compared with molecular simulations of a model electrode at corresponding surface charge densities. The simulation results are able to predict the experimental trends of the nonlinear relationship between the volumetric charge density and the applied voltage and the decrease of capacitance with increase of voltage.

The molecular simulations give qualitative and quantitative insight of the ion packing inside the pores and can help with the understanding of the nature of the change of capacitance and charge with the increase of the applied voltage observed experimentally. Namely, it is shown that the different pore sizes contribute differently to the capacitance and total charge adsorbed in the pores and demonstrate the selective absorption of cations in the pores. $\mathrm{EMIM}^{+}$is mainly occupying the micropores, where only at high voltages $\mathrm{TMA}^{+}$is able to enter them, which is not apparent when only the size of the ions is taken into account. The more flexible $\mathrm{EMIM}^{+}$cation can easily enter the smaller pores and adapt better to the pore geometry and curvature, which leads to a more effective packing of the smaller volume of these pores. This is also suggested from the umbrella sampling simulations where lower energy is needed for $\mathrm{EMIM}^{+}$to enter the micropore at low surface charge. These effects are not dominant in the mesopores where the more rigid and effectively smaller $\mathrm{TMA}^{+}$ions are able to enter the pore and contribute to the increase of the capacitance.

Mixed ionic liquid electrolytes have not been widely studied, although it has been shown that they can increase the capacitance compared to the neat IL. The complex interactions arising between different molecules in the mixed IL electrolyte can change the charge densities inside the pores and thus affect the capacitance. Here, we have shown the microscopic origin for this change in the mixed IL through a detailed analysis of our MD simulations, which has not been previously done, to the best of the authors' knowledge. Therefore, we hope that our MD simulations can provide a valuable insight into mixed IL supercapacitors and can help to design new electrolytes and devises with improved properties.

\section{Data Availability}

All data needed to evaluate the conclusions in the paper are present in the paper and the Supplementary Materials. Additional data related to this paper may be requested from the corresponding authors.

\section{Disclosure}

Aleksandar Y. Mehandzhiyski's current address is Laboratory of Organic Electronics, ITN, Linköping University, 60174 Norrköping, Sweden. Candy Anquetil-Deck's current address is Norwegian University of Science and Technology, Department of Energy and Process Engineering, Høgskoleringen 5, N-7491 Trondheim, Norway.

\section{Conflicts of Interest}

The authors declare that there is no conflict of interest regarding the publication of this article.

\section{Authors' Contributions}

B.A.G. and D.C. initiated and guided the research and reviewed and edited the paper. A.Y.M. performed and analyzed the MD simulations and prepared the manuscript. X.W. performed the experimental measurements, analyzed the data, and prepared the manuscript. C.A-D. prepared the manuscript.

\section{Acknowledgments}

The authors acknowledge the Research Council of Norway for the financial support (project no. 262399). The simulations were performed on resources provided by UNINETT Sigma2-the National Infrastructure for High Performance Computing and Data Storage in Norway.

\section{Supplementary Materials}

Supplementary Materials Calculation of the differential capacitance from the molecular dynamics simulations. (Supplementary Materials)

\section{References}

[1] X. Wang, M. Salari, D.-e. Jiang et al., "Electrode material-ionic liquid coupling for electrochemical energy storage," Nature Review Materials, vol. 5, no. 11, pp. 787-808, 2020.

[2] Poonam, K. Sharma, A. Arora, and S. K. Tripathi, "Review of supercapacitors: materials and devices," Journal of Energy Storage, vol. 21, pp. 801-825, 2019.

[3] S. Najib and E. Erdem, "Current progress achieved in novel materials for supercapacitor elec-trodes: mini review," Nanoscale Advances, vol. 1, no. 8, pp. 2817-2827, 2019.

[4] A. Izadi-Najafabadi, T. Yamada, D. N. Futaba, H. Hatori, S. Iijima, and K. Hata, "Impact of cell-voltage on energy and power performance of supercapacitors with single-walled carbon nanotube electrodes," Electrochemistry Communications, vol. 12, no. 12, pp. 1678-1681, 2010.

[5] A. Szewczyk, J. Sikula, V. Sedlakova, J. Majzner, P. Sedlak, and T. Kuparowitz, "Voltage dependence of supercapacitor capacitance," Metrology and Measurement System, vol. 23, no. 3, pp. 403-411, 2016.

[6] X. Wang, H. Zhou, E. Sheridan, J. C. Walmsley, D. Ren, and D. Chen, "Geometrically confined favourable ion packing for high gravimetric capacitance in carbon-ionic liquid 
supercapacitors," Energy \& Environmental Science, vol. 9, no. 1, pp. 232-239, 2016.

[7] X. Wang, A. Y. Mehandzhiyski, B. Arstad et al., "Selective charging behavior in an ionic mixture electrolytesupercapacitor system for higher energy and power," Journal of the American Chemical Society, vol. 139, no. 51, pp. 18681-18687, 2017.

[8] R. Wen, B. Rahn, and O. M. Magnussen, "Potential-dependent adlayer structure and dynamics at the ionic liquid/au(111) interface: a molecular-scale in situ video-stm study," Ange wandte Chemie International Edition, vol. 54, no. 20, pp. 6062-6066, 2015.

[9] E. Paek, A. J. Pak, and G. S. Hwang, "Erratum: A computational study of the interfacial structure and capacitance of graphene in [BMIM][PF6] ionic liquid [j. electrochem. soc., 160, a1 (2013)]," Journal of the Electrochemical Society, vol. 161, no. 10, pp. X15-X15, 2014.

[10] S. A. Kislenko, I. S. Samoylov, and R. H. Amirov, "Molecular dynamics simulation of the electrochemical interface between a graphite surface and the ionic liquid [bmim][pf6]," Physical Chemistry Chemical Physics, vol. 11, pp. 5584-5590, 2009.

[11] Y. Shim, Y. Jung, and H. J. Kim, "Graphene-based supercapacitors: a computer simulation study," The Journal of Physical Chemistry C, vol. 115, no. 47, pp. 23574-23583, 2011.

[12] C. Merlet, C. P'ean, B. Rotenberg, P. A. Madden, P. Simon, and M. Salanne, "Simulating supercapacitors: can we model electrodes as constant charge surfaces?," The Journal of Physical Chemistry Letters, vol. 4, no. 2, pp. 264-268, 2013.

[13] Y. Shim and H. J. Kim, "Nanoporous carbon supercapacitors in an ionic liquid: a computer simulation study," ACS Nano, vol. 4, no. 4, pp. 2345-2355, 2010.

[14] Q. Dou, M. Sha, H. Fu, and G. Wu, "Melting transition of ionic liquid [bmim][pf6] crystal confined in nanopores: a molecular dynamics simulation," The Journal of Physical Chemistry C, vol. 115, no. 39, pp. 18946-18951, 2011.

[15] G. Feng, S. Li, J. S. Atchison, V. Presser, and P. T. Cummings, "Molecular insights into carbon nanotube supercapacitors: capacitance independent of voltage and temperature," The Journal of Physical Chemistry C, vol. 117, no. 18, pp. 91789186, 2013.

[16] C. Merlet, B. Rotenberg, P. A. Madden et al., "On the molecular origin of supercapacitance in nanoporous carbon electrodes," Nature Materials, vol. 11, no. 4, pp. 306-310, 2012.

[17] M. Shamsipura, A. A. M. Beigi, M. Teymouri, S. M. Pourmortazavi, and M. Irandoust, "Physical and electrochemical properties of ionic liquids 1-ethyl-3-methylimidazolium tetrafluoroborate, 1-butyl-3-methylimidazolium trifluoromethanesulfonate and 1-butyl-1-methylpyrrolidinium bis(trifluoromethylsulfonyl)imide," Journal of Molecular Liquids, vol. 157, no. 1, pp. 43-50, 2010.

[18] W. Humphrey, A. Dalke, and K. Schulten, "VMD: visual molecular dynamics," Journal of Molecular Graphics, vol. 14, no. 1, pp. 33-38, 1996.

[19] H. J. C. Berendsen, J. P. M. Postma, W. F. van Gunsteren, A. DiNola, and J. R. Haak, "Molecular dynamics with coupling to an external bath," The Journal of Chemical Physics, vol. 81, no. 8, pp. 3684-3690, 1984.

[20] S. Tsuzuki, W. Shinoda, H. Saito, M. Mikami, H. Tokuda, and M. Watanabe, "Molecular dynamics simulations of ionic liquids: cation and anion dependence of self-diffusion coefficients of ions," The Journal of Physical Chemistry B, vol. 113, no. 31, pp. 10641-10649, 2009.

[21] J. N. Canongia Lopes, J. Deschamps, and A. A. H. P'adua, "Modeling ionic liquids using a systematic all-atom force field," The Journal of Physical Chemistry B, vol. 108, no. 6, pp. 2038-2047, 2004.

[22] J. N. A. Canongia Lopes and A. A. H. P'adua, "Nanostructural organization in ionic liquids," 536 The Journal of Physical Chemistry B, vol. 110, no. 7, pp. 3330-3335, 2006.

[23] G. Bussi, D. Donadio, and M. Parrinello, "Canonical sampling through velocity rescaling," The Journal of Chemical Physics, vol. 126, no. 1, article 014101, 2007.

[24] M. James, A. T. Murtola, R. Schulz et al., "Gromacs: high performance molecular simulations through multi-level parallelism from laptops to supercomputers," SoftwareX, vol. 1, pp. 19-25, 2015.

[25] C. Largeot, C. Portet, J. Chmiola, P.-L. Taberna, Y. Gogotsi, and P. Simon, "Relation between the ion size and pore size for an electric double-layer capacitor," Journal of the American Chemical Society, vol. 130, no. 9, pp. 2730-2731, 2008.

[26] X. Mao, P. Brown, C. Cervinka et al., "Self-assembled nanostructures in ionic liquids facilitate charge storage at electrified interfaces," Nature Materials, vol. 18, no. 12, pp. 1350-1357, 2020.

[27] G. M. Torrie and J. P. Valleau, "Monte Carlo study of a phaseseparating liquid mixture by umbrella sampling," The Journal of Chemical Physics, vol. 66, no. 4, pp. 1402-1408, 1977.

[28] D. Frenkel and B. Smit, "1," in Understanding Molecular Simulation: From Algorithms to Applications, Second, ser. Computational Science Series, Academic Press, San Diego, CA, USA, 2002.

[29] G. M. Torrie and J. P. Valleau, "Nonphysical sampling distributions in Monte Carlo free-energy estimation: umbrella sampling," Journal of Computational Physics, vol. 23, no. 2, pp. 187-199, 1977.

[30] D. A. Kofke and P. T. Cummings, "Quantitative comparison and optimization of methods for evaluating the chemical potential by molecular simulation," Molecular Physics, vol. 92, no. 6, pp. 973-996, 1997.

[31] S. Kumar, J. M. Rosenberg, D. Bouzida, R. H. Swendsen, and P. A. Kollman, "The weighted histogram analysis method for free-energy calculations on biomolecules. i. the method," Journal of Computational Chemistry, vol. 13, no. 8, pp. 1011-1021, 1992.

[32] B. Roux, "The calculation of the potential of mean force using computer simulations," Computer Physics Communications, vol. 91, no. 1-3, pp. 275-282, 1995. 